\title{
Grado de alfabetización académica y prácticas escritoras en el marco de un programa formativo
}

\author{
Degree of academic literacy and writing practices in the framework of a degree pro- \\ gram
}

\author{
Alba Naroa Romero González \\ e-mail: alromer@opendeusto.es \\ Universidad de Deusto. España
}

\author{
Manuela de las Nieves Álvarez Álvarez \\ e-mail: manuela.alvarez@deusto.es \\ Universidad de Deusto. España
}

\begin{abstract}
Resumen
La alfabetización académica de los estudiantes de grado es una cuestión que preocupa a los docentes universitarios e investigadores del ámbito nacional e internacional. En el contexto español todavía hay pocos estudios empíricos acerca de la naturaleza de las dificultades que experimentan los estudiantes y de cómo estas se transforman durante la trayectoria de los estudiantes en todo un programa formativo. Ahí se sitúa este estudio que tiene por objetivo conocer el grado de alfabetización académica de los estudiantes del grado en Lenguas Modernas y las prácticas escritoras que realizan. Para ello participan, por una parte, 96 estudiantes de los cuatro cursos con una reseña académica producida ad hoc, cuyo análisis se centra en la selección y organización de la información, la configuración de la voz del autor y los aspectos formales; y, por otra parte, participan 28 docentes del mismo grado con una entrevista que se centra en conocer las prácticas escritoras que solicitan a los estudiantes. Entre los resultados más destacados se constata que las prácticas escritoras que los estudiantes realizan en este programa formativo son, en general, poco exigentes cognitiva, retórica y discursivamente y, consecuentemente, no conducen a los estudiantes al desarrollo eficaz de la práctica escritora solicitada a efectos de este estudio.
\end{abstract}

Palabras clave: enseñanza superior; estudiante universitario; alfabetización; expresión escrita.

\begin{abstract}
Students' academic literacy is a question that concerns university professors and researchers at national and international level. In the Spanish context, there are still few empirical studies about the nature of the difficulties students experience and how those difficulties are transformed during the trajectory of the students along the whole degree. This is the framework for this study, which aims to know the level of academic literacy of the students of the degree in Modern Languages and the writing practices they perform. For that, on the one hand, 96 students from the four courses of the degree produce an academic review ad hoc. The analysis of this text focuses on the selection and organization of information, the configuration of the author's voice and formal aspects. On the other hand, 28 professors of the same degree participate with an interview that identifies the writing practices they request from students. Among the most outstanding results, it is noted that the writing practices that students perform in this degree are, in general, not very demanding cognitively, rhetorically and discursively; and, consequently, students do not develop effectively the requested writing practice for the purposes of this study.
\end{abstract}

Keywords: university education; university student; literacy; writing composition.

Recibido / Received: 12-01-2020

Aceptado / Accepted: 09-03-2020

Publicado en línea / Published online: 01-07-2020

Cómo referenciar este artículo / How to reference this article:

Romero González, A. N., \& Álvarez Álvarez, M. N. (2020). Grado de alfabetización académica y prácticas escritoras en el marco de un programa formativo. Tendencias Pedagógicas, 36, pp. 74-90. doi: $10.15366 /$ tp2020.36.06 


\section{Introducción}

La alfabetización académica de los estudiantes de grado es un tema que interesa a docentes universitarios e investigadores de todo el mundo. Ese interés, que nace de las dificultadas observadas en las producciones escritas que presentan los estudiantes universitarios, da lugar a dos movimientos preocupados por la enseñanza de la escritura en diferentes momentos y en diferentes contextos (Bazerman, Little, Bethel, Chavkin, Fouquette, \& Garufis, 2005; Russell, Lea, Parker, Street, \& Donahue, 2009).

El primero de estos, conocido como Writing Across the Curriculum (WAC), tiene lugar fundamentalmente en Estados Unidos en la primera mitad del siglo XX, aunque se extiende más allá de sus fronteras de manera más moderada. Inicialmente se plantea como un programa de enseñanza de habilidades discursivas y lingüísticas de carácter general en niveles universitarios, pero, con el paso del tiempo y la investigación científica, su orientación empieza a cambiar para plantear la enseñanza de la escritura académica de manera contextualizada en las disciplinas (Bazerman et al., 2005).

A partir de la segunda mitad del siglo XX, al otro lado del Atlántico, siendo Reino Unido el foco principal, surge el movimiento Academic Literacies (ACLITS), más conocido como Alfabetizaciones Académicas en el mundo hispano, el cual presenta en sus primeros estadios las mismas limitaciones que el WAC en lo referido a la enseñanza de la escritura académica (Russell et al., 2009). En las últimas décadas del siglo XX, con la aportación de figuras representativas como Street (1996) e Ivanic (1998), desde el ACLITS se empieza a teorizar en torno a la escritura académica como una práctica contextualizada, social y situada, la cual no está sujeta a unas normas formales generalizables a todas las disciplinas, sino que debe estar al servicio de las necesidades sociales y discursivas de cada disciplina.

En el marco de ambos movimientos se lleva a cabo toda una labor investigadora a lo largo del siglo XX desde diferentes orientaciones entre las que destacan las aportaciones de las teorías de la cognición (Bereiter \& Scardamalia, 1987; Flower \& Hayes, 1977), el enfoque sociocognitivo (Ortíz, 2011), el enfoque sociológico (Camps \& Millian, 2000), la antropología (Hymes, 1972), la pragmática (Chartier, 2004) y el análisis del discurso (Taylor, 2013). Esta labor investigadora se extiende hasta el siglo XXI bajo la perspectiva de los Nuevos Estudios de Literacidad, la cual comprende todos los estudios sobre las dificultades que tienen los estudiantes para producir los textos que los docentes les solicitan (Alter \& Adkins, 2006; Álvarez \& Boillos, 2015; Carlino, 2003; Figueroa \& Simón, 2011; Tapia, Burdiles, \& Arancibia, 2003).

La aportación de la investigación y los nuevos contextos sociales y educativos del siglo XXI conducen a que, desde el Espacio Europeo de Educación Superior (EEES), se considere la competencia comunicativa como una competencia esencial, eje de la Educación Superior, para el desarrollo de los estudios universitarios y el ejercicio profesional y, por consiguiente, una competencia muy presente en los nuevos planes de estudio que se adaptan a sus directrices (Cazorla, 2011; Ezeiza, 2012).

En concreto, en el contexto español se produce una reforma integral de las titulaciones de grado, máster y doctorado (Guzmán-Simón \& García-Jiménez, 2017) durante la primera década del siglo XXI. En este nuevo marco en el que la competencia comunicativa pasa a cobrar una importancia notable, se constatan dificultades diversas en el grado de alfabetización académica de los estudiantes universitarios. Estas dificultades se sitúan tanto en aspectos diferentes de los textos académicos producidos (Álvarez \& Boillos, 2015; Castelló, 2009, 2015; Guzmán-Simón \& García-Jiménez, 2015, 2017) como en aspectos relativos al proceso completo de la elaboración de los mismos (postergar el momento de empezar a escribir; dificultades para acotar el tema, seleccionar la información u organizar las ideas; revisión superficial del texto, entre otros), y de cuya naturaleza se conoce poco (Camps \& Castelló, 2013). Además, son escasos los estudios acerca de cómo evoluciona el proceso de alfabetización académica de los estudiantes dentro de un programa formativo (Camps \& Castelló, 2013; Chois \& Jaramillo, 2016).

Ese es el marco en el que se sitúa este estudio empírico de tipo transversal que analiza el grado de alfabetización académica de los estudiantes de los cuatro cursos del grado en Lenguas Modernas (Universidad de Deusto) y que identifica las prácticas escritoras previas a la escritura del trabajo de fin de grado (TFG) y realizadas a lo largo de ese período formativo. Esto se aborda desde los textos producidos por los estudiantes y desde entrevistas a docentes para dar respuesta a los siguientes objetivos:

- Analizar qué información seleccionan y cómo la organizan para la construcción de un texto. 
- Analizar cómo configuran la voz como autores académicos.

- Examinar qué uso realizan de los aspectos formales del texto.

- Identificar qué prácticas escritoras realizan en el grado según el curso.

El artículo se organiza en cuatro apartados bien diferenciados. Después de este apartado introductorio, se sigue con la indicación de diferentes aspectos del marco metodológico como los participantes, el instrumento de obtención de datos o el proceso de análisis. A continuación, se presentan los resultados y la discusión que de estos se deriva; y, para terminar, se ofrecen las conclusiones del estudio.

\section{Marco metodológico}

\subsection{Participantes}

Los participantes de este estudio son, por una parte, los 96 estudiantes de nueva matrícula (no han repetido ni realizado previamente otros grados) del grado en Lenguas Modernas (LLMM) de la Universidad de Deusto (Bilbao) durante el curso académico 2015/2016. Por otra parte, participa toda la población de docentes (28 en total) del mismo grado.

\subsection{Instrumento de obtención de datos}

\subsubsection{Reseña académica de formación}

La obtención de datos se lleva a cabo mediante una reseña académica de formación, género académico que se ha vuelto más frecuente entre las prácticas escritoras que deben producir los estudiantes de grado (Alzari, D’Alessandro, \& Radiminski, 2014) debido a su reciente caracterización como una herramienta útil para favorecer su aprendizaje al exigirles habilidades de interpretación y análisis crítico (Hyland, 2007). Esta se define como un texto académico que tiene la función de informar sobre una referencia bibliográfica, emitir juicios de valor sobre diferentes aspectos de la misma e influir en el lector. Para ello, aunque no hay una estructura propiamente fijada, se distinguen hasta cuatro propósitos comunicativos en su elaboración: presentar el libro, describir el libro, evaluar el libro y recomendar el libro (Navarro \& Abramovich, 2012; Alzari, 2012; Alzari, D’Alessandro, \& Radiminski, 2014).

Este género resulta especialmente idóneo para la recogida de datos en este estudio por varias razones: es un género académico reconocido; supone el uso de dispositivos discursivos complejos como la presentación, la descripción, la evaluación, etc.; exige del autor una selección consciente de los contenidos; es un texto conocido por los estudiantes de grado; es un texto breve, lo cual facilita su aplicación; y es un género fácil de acomodar a diferentes niveles de un mismo grado para que los estudiantes puedan producirlo en igualdad de condiciones.

Para la producción de esta reseña se selecciona el manual de Gramática didáctica del español escrito por Leonardo Gómez Torrego (2011) debido a la familiaridad de la temática del libro con los intereses de los estudiantes del grado, y por la portada, contraportada, índice y presentación del libro, cuya configuración permiten que el estudiante produzca la reseña de manera espontánea. Tras la elección de este manual, se crea una situación comunicativa verosímil que implica la producción de una reseña académica de formación por parte de los estudiantes: la biblioteca de la universidad acaba de incorporar esta obra en el catálogo y necesita una reseña de la misma.

Antes de aplicar el instrumento con los participantes del estudio, se realiza un pretest con los estudiantes del grado en Lenguas Modernas y Gestión que conduce a que se realicen modificaciones en la instrucción que finalmente se aplica.

\subsubsection{Entrevista}

Se diseña una entrevista semiestructurada compuesta por 12 cuestiones en torno a la alfabetización académica de los estudiantes, las prácticas escritoras que llevan a cabo los estudiantes, la metodología, etc.

El instrumento se valida a partir de un juicio de expertos en el que participan cinco doctores especialistas en creación de instrumentos como la entrevista que, individualmente, realizan sugerencias y 
evaluaciones que sirven para realizar correcciones referidas, fundamentalmente, a aspectos relacionados con la comprensión del interrogante. Posteriormente, se realiza el pilotaje con una docente voluntaria de la facultad de Ciencias Sociales y Humanas, cuya participación permite realizar ajustes y reformular algunas expresiones.

\subsection{Proceso de análisis}

El proceso de análisis tiene lugar en cuatro fases. En una primera fase del análisis, se atiende a la selección y la organización de la información, a cómo se construye el texto, asumiendo el modelo de análisis de género iniciado por Swales $(1990 ; 2004)$. En este análisis son especialmente útiles los conceptos de movimiento retórico (MR), secuenciación discursiva, pasos y subpasos (la información y las ideas) que se despliegan en cada MR y, finalmente, la densidad informativa y espacio dedicado a cada MR.

A partir de esta teorización, elaboramos y validamos un modelo de análisis retórico-discursivo de las reseñas producidas por estudiantes de grado (Romero, 2019), modelo aplicado en este estudio y que plantea que la reseña académica de formación se construye a partir de dos movimientos retóricos (describir y valorar) que se concretan en una sucesión de pasos y subpasos.

En una segunda fase del análisis, se atiende a cómo configuran la voz del autor. Para ese propósito, se adopta el modelo analítico de Hyland (2007) del metadiscurso, que entiende el texto como un medio de comunicación social mediante el cual el autor trata de influir en el lector. Este modelo permite analizar aspectos de la voz del autor como su presencia explícita en el texto (las huellas que deja de su figura), su posicionamiento respecto a los contenidos (grado de implicación del autor; énfasis o intensificación de su compromiso con las ideas expresadas o, por el contrario, elusión del compromiso), la interacción con el lector o con otras voces (intertextualidad) y los elementos textuales (conectores de tipo lógico, marcadores discursivos, marcadores de endofórica, glosas de código para ejemplificar o explicar) que sirven para orientar al lector en la lectura.

En la tercera fase del análisis, se identifican y clasifican los usos incorrectos que realizan los estudiantes de los aspectos formales del texto como la ortografía, la gramática o la puntuación según la normativa de la Real Academia de la Lengua (2010a; 2010b).

En la cuarta y última fase, se identifican las prácticas escritoras que los estudiantes realizan en el grado y se clasifican según el curso.

\section{Resultados y discusión}

En este apartado los resultados y la discusión se organizan en torno a los objetivos del estudio.

\subsection{Selección de la información en la elaboración de la reseña}

La selección de la información que realizan los estudiantes para la construcción de la reseña varía según el curso y atiende a dos objetivos comunicativos: describir y valorar. En la tabla 1 se presenta la información a la que recurren y el porcentaje de frecuencia que alcanza cuando están describiendo en los cuatro cursos. La figura 1 representa la trayectoria de cómo evoluciona dicha selección informativa a lo largo de los mismos.

Tabla 1.

Selección de la información según el curso

\begin{tabular}{lllll}
\hline & Primero & Segundo & Tercero & Cuarto \\
\hline Autor & $100 \%$ & $95,4 \%$ & $100 \%$ & $77,7 \%$ \\
Otras características & $85,3 \%$ & $77,2 \%$ & $53,8 \%$ & $62,9 \%$ \\
Tema & $55,8 \%$ & $54,5 \%$ & $38,4 \%$ & $59,2 \%$ \\
Organización del libro & $47 \%$ & $63,6 \%$ & $92,3 \%$ & $81,4 \%$ \\
Enfoque teórico & $11,7 \%$ & $13,6 \%$ & $23 \%$ & $72,9 \%$ \\
Objetivo & $32,3 \%$ & $36,7 \%$ & $30,7 \%$ & $14,8 \%$ \\
Contexto de producción & $35,3 \%$ & $22,7 \%$ & $15,3 \%$ & $7,4 \%$ \\
& & & &
\end{tabular}




\begin{tabular}{lllll} 
Localización & $0 \%$ & $4,5 \%$ & $0 \%$ & $3,7 \%$ \\
Tipo de libro & $0 \%$ & $0 \%$ & $0 \%$ & $3,7 \%$ \\
\hline
\end{tabular}

Fuente: elaboración propia.

Figura 1.

Evolución del uso de la información para describir según el curso

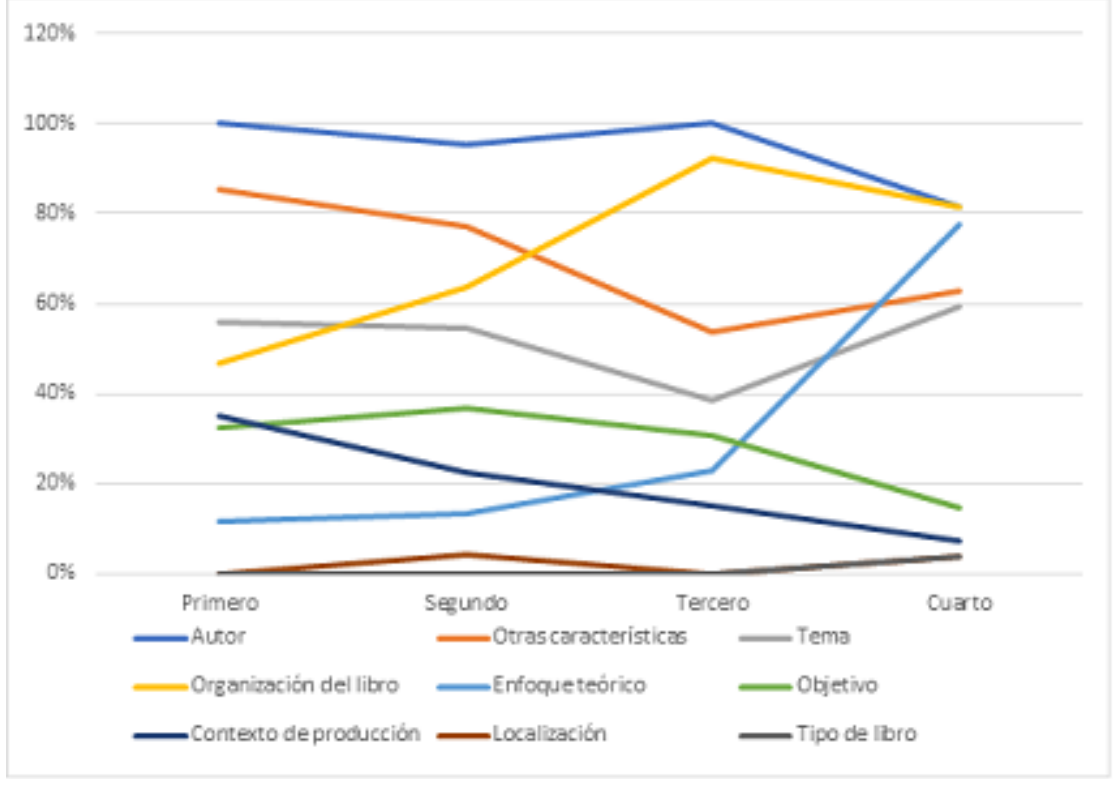

Fuente: elaboración propia.

Como se puede observar en la tabla 1 y en la figura 1, la selección de la información que realizan varía de un curso a otro de manera notoria. Mientras que los estudiantes de los dos primeros cursos se refieren fundamentalmente a los rasgos más externos de la obra, como puede ser presentar al autor y exponer otras características del libro relacionadas con su diseño, los estudiantes de tercero y, sobre todo, de cuarto realizan una selección más pertinente que prioriza la organización de los contenidos del libro sobre otro tipo de información. Cabe destacar en esta evolución de un curso a otro la consolidación del enfoque teórico como una información de gran valor en cuarto, pues su recurrencia supone que los estudiantes ya no comprenden el conocimiento como homogéneo, sino problematizado.

Algunos ejemplos ilustrativos de esta selección de la información:

«(1) El libro «la gramática didáctica del español [sic]» está escrito por Leonardo Gómez Torrego que es un doctor en filología [sic] y director del CSIC.

(Informante 1.1)»

«(2) ... pertenece a la editorial SM. A parte [sic] de Leonardo Torrego (quien ha sido el autor del libro), hay también más gente que ha colaborado en la edición y supervisión como es el ejemplo de Concepción Maldonado, la cual es Doctora [sic] en Filología Hispánica.

No es la primera edición que se hace de este libro, la versión actual está corregida y aumentada. (Informante 1.25)»

«(3) Este libro, «Gramática didáctica del español», ha sido escrito por el Doctor [sic] en filología [sic] Leonardo Gómez Torrego, con el objetivo de facilitar el entendimiento del español y su gramática.

(Informante 2.53)»

«(4) ... tiene como tema principal explicar la gramática más didáctica resolviendo, de ese modo dudas gramaticales.

(Informante 2.43)» 
«(5) La organización de los contenidos está dividida en capítulos que siguen un orden en función a su grado de dificultad. En primer lugar, encontramos una breve introducción que precede a los capítulos. Tras la introducción, se abordarán las clases de palabras en el primer apartado, donde podremos examinar el sustantivo, el adjetivo, los determinativos, los pronombres, los verbos, los adverbios, las preposiciones, las conjunciones y las interjecciones. El segundo capítulo, cuya extensión es más limitada, estará dedicado a las oraciones, los enunciados y los grupos sintácticos. Dicho apartado estará segmentado en tres subapartados: el primero estará dedicado a los enunciados y las oraciones, el segundo a los grupos sintácticos y el tercero a oraciones y conjuntos. Los últimos dos capítulos, fonética y fonología; y ortografía, estan [sic] estrechamente relacionados y estaran [sic] orientados hacia las partes de la gramática que suelen entrañar mayor dificultad.

(Informante 3.66)»

«(6) Esta gramática presenta un enfoque descriptivo y normativo...

(Informante 4.72)»

«(7) Cada unidad contiene explicaciones de todo aquello que se debiera transmitir desde un libro de gramática. Desde lo más básico hasta lo más complejo y avanzado. Contiene, además, ejercicios con respuestas y aclaraciones para poner en práctica todo aquello que el libro transmite.

(Informante 4.75)»

No parece constatarse una evolución similar en la selección de la información cuando los estudiantes valoran, como se observa en la tabla 2 y en la figura 2.

Tabla 2.

Selección de la información para valorar según el curso

\begin{tabular}{lllll}
\hline & Primero & Segundo & Tercero & Cuarto \\
\hline Destinatario ideal & $82,3 \%$ & $59 \%$ & $100 \%$ & $81,4 \%$ \\
Juicios de valor positivos o negativos & $67,6 \%$ & $45,4 \%$ & $76,9 \%$ & $44,4 \%$ \\
Recomendación & $20,5 \%$ & $4,5 \%$ & $0 \%$ & $0 \%$ \\
Originalidad & $5,8 \%$ & $4,5 \%$ & $0 \%$ & $14,8 \%$ \\
\hline
\end{tabular}

Fuente: elaboración propia.

Figura 2.

Evolución del uso de la información para valorar según el curso

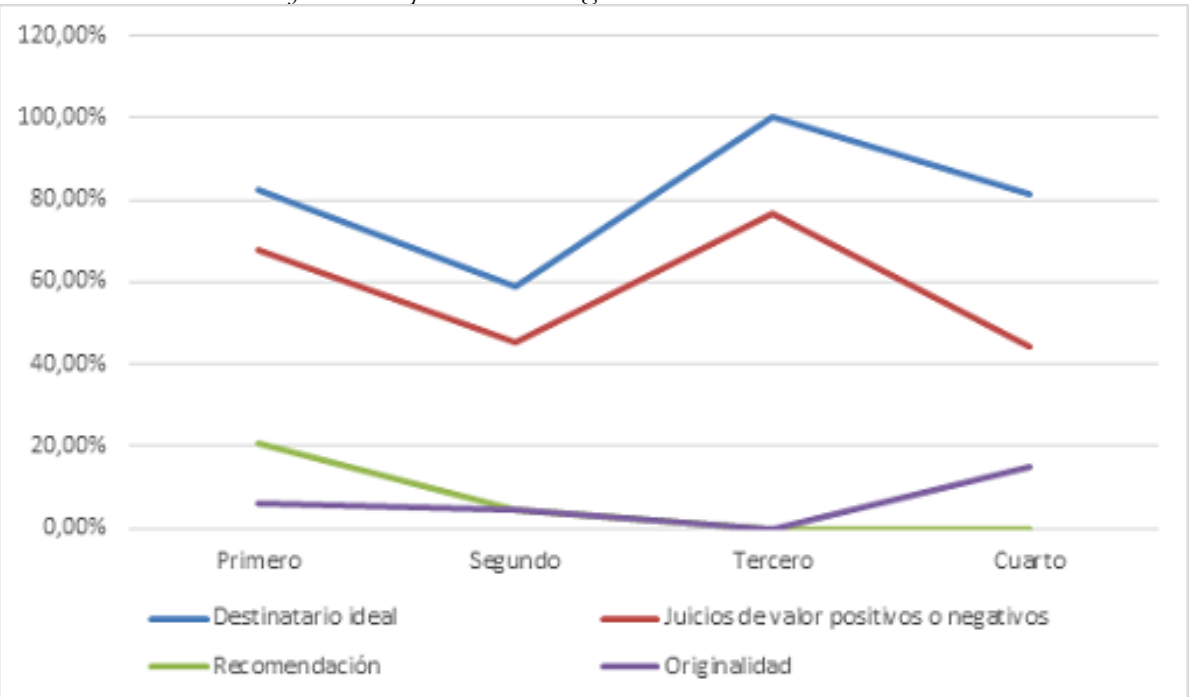

Fuente: elaboración propia.

No se registran cambios sustanciales en cuanto a la selección de la información para valorar el libro de un curso a otro. Lo más llamativo es que, por un lado, las informaciones relativas a la recomendación del libro y la valoración de su originalidad prácticamente desaparecen y que, por otro lado, los 
juicios de valor son mucho menos frecuentes en cuarto que en primero. Asimismo, estos últimos suelen estar destinados a aspectos marginales del texto que no son significativos para la tarea comunicativa planteada y a la que da respuesta la reseña.

Algunos ejemplos ilustrativos de esta selección de la información cuando los estudiantes valoran:

«(8) En conclusión, he de decir que esta obra es una gran aportación para la lengua castellana y podrá facilmente [sic] ser usada en cualquier centro académico ya que es de ayuda e indaga bastante bien en [sic] casi todos los temas importantes de nuestro idioma.

(Informante 1.26)»

«(9) Sin embargo, tal como se dice en el mismo libro, el lector no debe esperar lo más extenso y a la vez concreto de las explicaciones, pues eso requeriría una gran profundización en cada apartado.

(Informante 2.47)»

«(10) Este libro esta [sic] dirigido a alumnos y profesores de secundaria, estudiantes de filología [sic] o para los amantes de la gramática española.

(Informante 1.7)»

«(11) Este libro es en muchos aspectos novedoso, ya que hace que el lector discurra y discuta el contenido.

(Informante 4.76)»

«(12) Es recomendable su lectura por el hecho de que profundiza sus explicaciones en áreas como las clases de palabras (sustantivo, adjetivo, verbo, adverbio...), las oraciones y grupos, la fonética y la fonología y la ortografía junto con la acentuación, puntuación, etc.

$(\ldots)$

Como se puede ver, el libro es bastante completo y nos permite entrar en diferentes y variadas áreas para resolver nuestras mayores dudas sobre el idioma, por lo que su uso y/o estudio son muy recomendables.

(Informante 1.13)»

Además de conocer la información a la que recurren los estudiantes por curso para describir y valorar, el análisis de la selección de la información también permite conocer la cantidad de ideas, de informaciones, que los estudiantes utilizan en cada caso. Esto revela una escasez informativa en todos los cursos que infiere que, si bien la selección de la información es cada vez más pertinente para describir, esta resulta insuficiente para completar el propósito comunicativo del texto.

En definitiva, aunque la reseña académica de formación es un género breve y sencillo, con el paso de los años los estudiantes no parecen abordarla en toda su complejidad. La selección de la información para describir es más pertinente en cuarto que en primero, pero sigue siendo limitada y poco variada, y no pone en relación los contenidos del texto con el área disciplinar.

\subsection{Organización de la información en la elaboración de la reseña}

La organización de la información, agrupada en los objetivos comunicativos de describir y valorar, sigue cuatro modelos de secuenciación discursiva diferentes.

Tabla 3.

Modelos de secuenciación discursiva.

\begin{tabular}{llll}
\hline Modelo 1 & Modelo 2 & Modelo 3 & Modelo 4 \\
\hline Describir+Valorar & Valorar+Describir & Describir & Valorar \\
\hline
\end{tabular}

Fuente: elaboración propia.

Los dos primeros modelos expuestos en la tabla 3, Modelo 1 y Modelo 2, se valen de los dos objetivos comunicativos, pero varían en la ordenación. Mientras que en el Modelo 1 primero se describe y luego se valora, en el Modelo 2 se empieza con la valoración seguida de la descripción. En ambos casos, esta secuenciación se puede presentar de manera recursiva, es decir, en textos en los que se describe, se valora, se vuelve a describir, a valorar, etc.; y supone una ruptura del discurso que es 
incoherente con lo expresado previamente o que está desencadenada, precisamente, por el enunciado anterior. Algunos ejemplos ilustrativos de este fenómeno:

«(13) Este libro como su título («La gramática y didáctica del español» [sic]) indica, trata sobre la gramática española. El autor del libro es Leonardo Gómez Torrego. Como he dicho, en este libro encontraremos apartados que nos hablarán sobre gramática, y didáctica española. En mi opinión el libro está bien organizado, ya que hay apartados separados en subapartados que son de gran ayuda. Dentro del libro, como apartados o temas más importantes encontraremos «Las clases de palabras», «Oraciones y grupos», «Fonética y Fonología» y «Ortografía» [sic]. (Informante 1.3)»

«(14) El autor de este libro es Leonardo Gómez Torrego, Doctor [sic] en Filología e investigador del CSIC. Es un libro que trata a gramática más didáctica, resuelve dudas con múltiples ejemplos y tiene un enfoque descriptivo y normativo, por lo que lo encontramos muy útil tanto para profesores como para alumnos.

(Informante 1.23)»

Los dos modelos restantes, Modelo 3 y Modelo 4, recurren a un único objetivo comunicativo, ya sea describir o valorar. Ejemplo ilustrativo del Modelo 3:

«(15) El libro que va a ser utilizado es «La gramatica [sic] didactica [sic]». Este está escrito por Leonardo Gómez Torrego. Este libro tiene explicado [sic] cosas como las clases de palabras, oraciones y grupos, fonetica [sic] y fonologia [sic] y la ortografia [sic]. En cada una vienen explicadas cada apartado explicando [sic] las cosas más importantes de cada grupo. Ej.: [sic] dentro de las clases de palabras, tenemos el sustantivo, adjetivos [sic], los determinativos, pronombres [sic], el verbo, el adverbio, las preposiciones y las conjunciones.

Deciros que este libro explica la gramatica [sic] de manera didactica [sic] por diferentes razones.

Se explican los terminos [sic] con claridad y de manera que sean faciles [sic] de entender.

Avisa en varias ocasiones de los fallos más comunes.

Nos explica de diferente manera algunos puntos.

Nos explica con diferentes nombres lo mismo porque no en todas partes se hace igual.

El diseño hace que sea facil [sic] de entender.

Después de cada capitulo [sic] tiene ejercicios y sus respuestas.

(Informante 2.35)»

Ejemplo ilustrativo del Modelo 4:

«(16) En este libro «La gramática didáctica del español» se hace una breve presentación, la cual, personalmente, la encuentro un tanto tosca, ya que da razones muy largas por la cual le acompaña el adjetivo didáctico a esta monografía. Aunque visualizando el índice del libro, es bastante completo. Este, podría interesarle a cualquier persona, desde estudiantes de cualquier carrera o escuela, hasta a una persona corriente, a la quien [sic] le surge una duda, y quiere resolverla. Ya que, no solo explica lo «raro» de la gramática española, y con raro me refiero, a eso que no todo el mundo sabe hacer, como analizar una frase sintácticamente. [sic] Sino que también explican [sic] conceptos sobre la ortografía, y, ¿quién no ha tenido alguna vez dudas de cómo se escribe una palabra? Yo creo que nadie.

Por ello, creo que aun siendo útil para informar a cualquier persona, destacaría más entre los estudiantes de Filología Hispánica, por ejemplo. Este, les daría información sobre toda duda que puedan tener acerca de la gramática, y sería el adecuado. Además, está perfectamente estructurado con un índice muy completo, por lo que fácilmente se encontraría lo que se está buscando. Así que, solo basándome en la portada, presentación e índice (que es lo dado) creo que es un libro muy bien planteado y que la gente al cogerlo, no dudaría en que es el libro más completo que podrían encontrar.

(Informante 2.37)»

La figura 3 presenta la utilización de estos modelos según el curso para la secuenciación discursiva de las reseñas. 
Figura 3.

Evolución de los modelos secuenciación discursiva según el curso

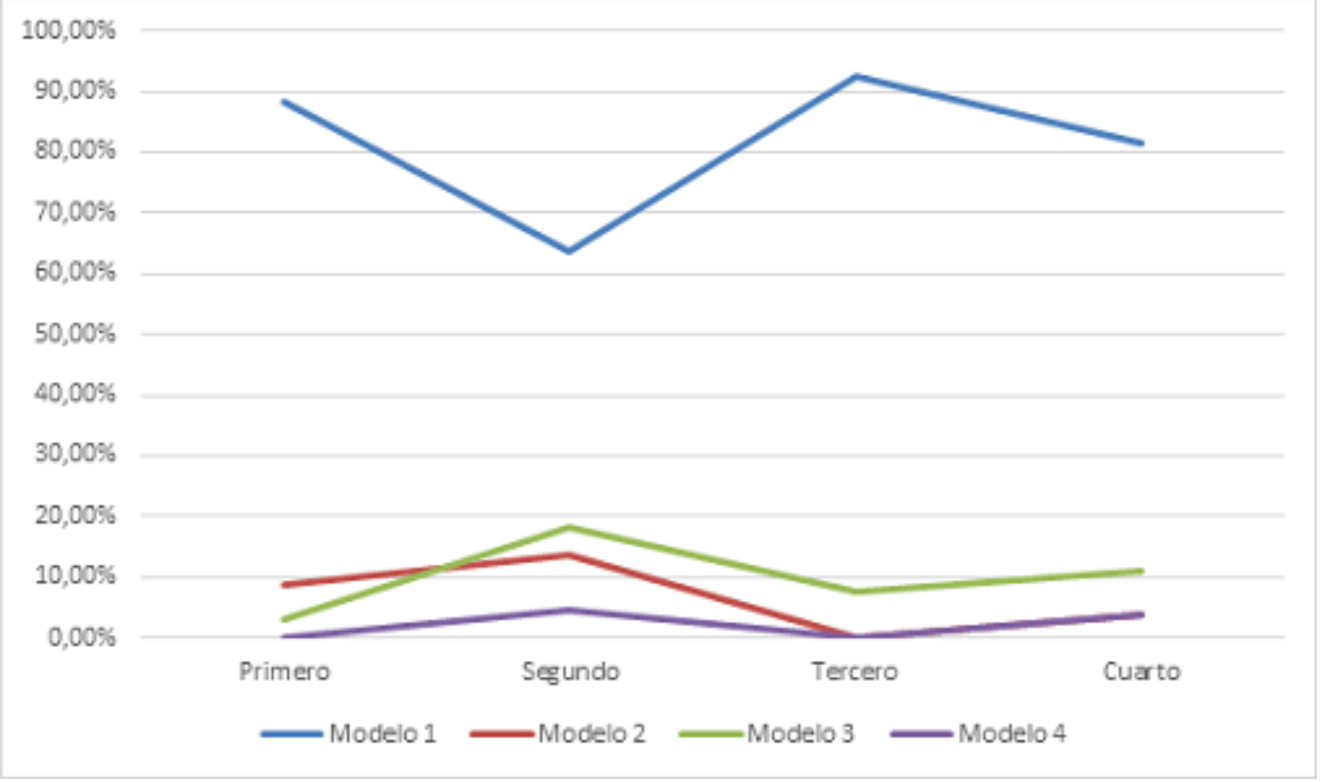

Fuente: elaboración propia.

El Modelo 1, aquel que responde de manera más ajustada al propósito comunicativo expuesto en la instrucción con la que se ha planteado primero la descripción seguida de la valoración, es también el más recurrente en todos los cursos frente a otros modelos organizativos más deficitarios que no tienen una presencia tan elevada. No obstante, cabe destacar en segundo y cuarto la fuerte presencia de otros modelos, especialmente de aquellos que solo responden a un objetivo comunicativo, pues es un modo de presentar la información que no contribuye al propósito comunicativo del texto.

En resumen, la información se organiza en todos los cursos, fundamentalmente, siguiendo el orden que se sugiere en la instrucción y aquel que se ajusta mejor a la estructura del género. Sin embargo, la constancia de textos en los que se presentan secuenciaciones discursivas en las que se describe y se valora de manera recursiva, produciéndose una ruptura del discurso, pone de manifiesto el desconocimiento por parte de los estudiantes de este género y una escasa habilidad en la progresión temática del texto. Asimismo, otras formas de secuenciación discursiva con una presencia menor tampoco responden a la estructura de la reseña.

\subsection{Configuración de la voz como escritores académicos}

En lo que respecta a la configuración de la voz del autor, se observa, por una parte, que el autor está muy presente en los textos del primer y segundo curso mediante la primera persona del singular. Esto contraviene los convencionalismos y especificidades del discurso académico español, según los cuales el autor no se hace visible en el texto (Goethals y Delbecque, 2001). Algunos ejemplos ilustrativos:

«(17) En mi opinión el libro está bien organizado, ya que hay apartados separados en subapartados que son de gran ayuda.

(Informante 1.3)»

«(18) Yo creo que nadie.

(Informante 2.37)»

En tercero y cuarto, en cambio, el autor ya no se hace visible a través de la primera persona, sino que se oculta por completo tras la tercera persona del singular, lo cual viene a coincidir con los modos de presentarse el autor en el discurso académico español.

Por otra parte, el posicionamiento del autor respecto a los contenidos es muy bajo en los dos primeros cursos y se vuelve más escaso a medida que avanzan los cursos. Ahondando en estos posicionamientos, se constata que los estudiantes tienen una manera de posicionarse desde primero que 
se centra únicamente en marcar la importancia de las ideas, la probabilidad de los juicios que se emiten o la certeza de algunas afirmaciones, sin mantener un hilo argumental que resulte persuasivo para el lector. En cuarto, estos posicionamientos son cada vez menos variados, al expresar únicamente la probabilidad de los juicios de valor emitidos por los estudiantes. Todo ello, además, recurriendo siempre a unas mismas expresiones extraídas del texto fuente que no permiten conocer la auténtica valoración de los estudiantes. Algunos ejemplos:

«(19) Este libro podría ser muy útil para estudiantes de carreras que tengan relación con el lenguaje así como estudiantes de Lenguas Modernas o de alguna Filología. (Informante 1.34)»

«(20) En definitiva, la gramatica [sic] didáctica del español, presenta muchos aspectos de novedad que lo [sic] hace, sin duda, más atractivo [sic] porque posiblemente el lector se vea obligado a discrepar o a discutir sobre algunos fenómenos concretos.

(Informante 1.26)»

«(21) Por todo lo mencionado anteriormente, es un libro totalmente recomendable, con el que aquel que quiera dominar la gramática de esta lengua podrá contar.

(Informante 1.14)»

Estos resultados sobre el posicionamiento respecto a los contenidos apuntan a un autor cada vez menos implicado con los contenidos que expresa, lo cual viene a coincidir con los resultados hallados por otros autores sobre textos producidos por estudiantes universitarios (Aguilar \& Fregoso, 2013; Castro \& Sánchez, 2013; Gil-Salom \& Soler-Monreal, 2014; Sánchez-Jiménez, 2017; Venegas, Meza, \& Martínez, 2013). No obstante, según ciertos estudios sobre esta misma cuestión (Castelló, Corcelles, Iñesta, Bañales, \& Vega, 2011; Colmenares, 2013), los estudiantes de grado debieran adquirir progresivamente habilidades para responsabilizarse y comprometerse con lo que escriben y para relacionar las ideas propias con el saber ajeno.

Ahondando en las características de la voz que construyen como autores académicos, se observa que el lector del texto parece estar muy presente en los textos de todos los cursos, con especial atención en tercero y cuarto. Estas interacciones con el lector se centran en la expresión del plural inclusivo en la desinencia verbal y en pronombres posesivos en posición de no sujeto. Por ejemplo:

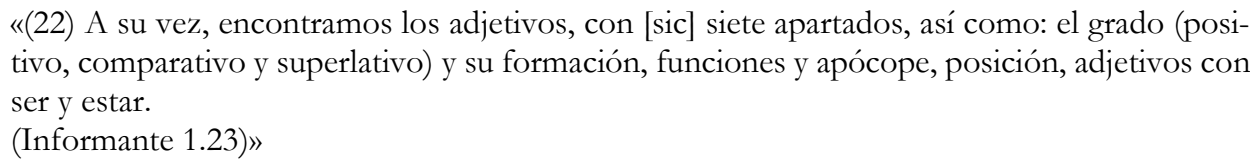

«(24) Hay que ir más allá para tener un perfecto manejo sobre la gramática ya sea de nuestra lengua materna o de otra lengua que se estudie.

(Informante 2.52)»

Como se puede apreciar en los ejemplos, estos modos de interactuar con el lector, si bien lo hacen partícipe de la lectura en algunos momentos, no resultan persuasivos porque no contextualizan la información en el marco disciplinar al que pertenecen para que el lector valore el interés de la lectura. Se trata, en todo caso, de una interacción más próxima a la oralidad. Estas mismas dificultades para conjugar adecuadamente la voz del autor del texto con la del lector y para contextualizar la obra en general también se observan en la no presencia de otras voces en las reseñas.

Finalmente, en esa creación de la voz como escritores académicos se observa que, en el uso de los elementos textuales que estructuran el texto para orientar al lector, los estudiantes establecen relaciones sintácticas de poca complejidad, principalmente aditivas y con poca frecuencia causales, que se expresan recurriendo a los mismos conectores (también, además, ya que). Esta simplicidad sintáctica se mantiene igual de primero a cuarto. Algunos ejemplos ilustrativos: 
«(25) Creo que puede ser de gran utilidad sobre todo para estudiantes de secundaria que necesiten refuerzo con la gramática, ya que dispone de muchos ejemplos y ejercicios con sus respectivas soluciones.

(Informante 1.7)»

«(26) Además, está dotado de prevenciones al lector contra confusiones y abundantes remisiones de unos lugares a otros. Por si esto fuera poco, ofrece innumerables correspondencias terminológicas e información sobre normativa.

(Informante 1.17)»

Asimismo, los estudiantes realizan una estructuración del texto mediante marcadores discursivos que solo se ocupa, en los pocos casos en los que se utiliza, de cerrar el discurso en los cuatro cursos, nuevamente recurriendo a las mismas expresiones (por último y finalmente).

«(27) Para acabar, el libro ofrece las soluciones de los ejercicios como una manera más práctica y rápida de trabajar con la teoría explicada a lo largo de todo el libro.

(Informante 2.51)»

«(28) Para finalizar el libro dispone de unas soluciones a los ejercicios y de un índice temático. (Informante 3.66)»

Estos resultados muestran que los estudiantes configuran una voz de sí mismos como escritores académicos que se caracteriza por ser cada vez más neutra y menos implicada con aquello que «se dice»; no comprometida con los contenidos expresados, ni situándolos en el marco del conocimiento disciplinar al que se refieren; además, no se establecen relaciones lógicas de carácter complejo entre las ideas, y no se materializa de manera clara la estructura del texto.

Estos mismos resultados se constatan en otros estudios sobre alfabetización académica inicial (Arrosi, Axelrud, D’Agostino, \& Eisner, 2003; Errázuriz \& Fuentes, 2010; Marinkovich \& Poblete, 2014), pero no debieran reflejarse en un estudio que sigue la trayectoria de unos estudiantes de primero a cuarto en un programa formativo.

\subsection{Aspectos formales del texto}

En el análisis de los textos se han constatado una serie de usos incorrectos de la ortografía asociados a la acentuación, el uso de las mayúsculas y los signos de puntuación que influyen negativamente en la calidad del texto.

En el caso de la acentuación, se observa que esta empeora en la trayectoria de los cuatro cursos, repitiéndose además los mismos errores en las mismas expresiones, las cuales aparecen además en el texto fuente para realizar la reseña y son propias del ámbito académico en el que se están formando. Aparecen sin acentuar palabras como: encontrarás, insertará, nos ayudará, fácil, didáctico, básicamente, términos, ortografía, sintáctico, difícil, lógico, párrafo, múltiple, fonética, está expuesto, partes de él, más, lingüística, etc.

En el uso de las mayúsculas también se repiten los mismos errores en las mismas expresiones en los cuatro cursos, pero, en este caso, no se produce un empeoramiento a medida que pasan los cursos. Estos se concentran en los títulos, como el del libro, o en la referencia a cargos o titulaciones (doctor o investigador).

En ambos aspectos, sin que pueda descartarse al menos en algunos casos, desconocimiento de la normativa, se infiere que los participantes del estudio no valoran la importancia del uso correcto de la ortografía en ninguno de los cursos, lo cual repercute negativamente en la comprensión del texto.

Por último, la puntuación también se presenta como un déficit en los textos que no desaparece en ninguno de los cursos; de hecho, parece empeorar, al igual que la acentuación. En este caso, las dificultades se concentran en el uso de la coma en la coordinación de elementos en una enumeración (esta obra es una gran aportación para la lengua castellana y podrá fácilmente ser usada en cualquier centro académico), en los cambios de orden (Gracias a esta forma innovadora de plasmar los contenidos es recomendado tanto como [sic] para estudiantes no universitarios), en el señalamiento de los incisos (La gramática didáctica del español es un libro, como bien dice el título sobre varios aspectos de la gramática escrita) y cuando separa sujeto y verbo (Leonardo Gómez. Torrego, ha querido indagar en las raíces de la gramática). 
Tampoco realizan un uso adecuado del punto y coma al ser sustituido por otros signos de puntuación como la coma y el punto y seguido (Son muchos los conocimientos que se dan por hecho que se controlany que se hace buen uso de ellos, por el contrario, en diversas ocasiones cometemos pequeños y grandes errores...).

El punto y aparte se utiliza para separar elementos de un mismo tema o, por el contrario, no se utiliza cuando es necesario separar diferentes temas. Por ejemplo:

«(29) Por último, cabe remarcar que también se trata de un libro muy dinámico, puesto que, además de contenido más teórico, se propone una serie de ejercicios y se aportan soluciones, de forma que lo aprendido se pueda usarse [sic] de forma práctica.

Es por ello, un libro útil, amplio y práctico, que puede servir de utilidad a personas interesadas en diversos aspectos de la gramática española.

(Informante 1.2)»

«(30) Este libro relacionado con la gramática didáctica española ha sido escrito por Leonardo Gómez Torrego. Se trata de un libro sencillo y útil para aquellos que tengan cierto interés en la gramática. Estando dividido en 5 capítulos, abarca temas como los sustantivos, adjetivos, determinativos, verbos, preposiciones, etc. También nos proporciona información sobre las oraciones, la fonética y la ortografía. Como ha sido anteriormente mencionado, es un libro sencillo y fácil de usar, ya que los contenidos se exponen de una manera simple y siempre acompañados de ejemplos y ejercicios, cuyas soluciones podemos encontrar al final. El escritor utiliza constantemente párrafos cortos para poder adquirir la información con mayor facilidad.

(Informante 4.86)»

Por último, el punto final es utilizado en párrafos que no tienen carácter conclusivo, como se puede observar a continuación:

«(31) Siendo un libro de gramática en palabras del autor «esencial», es posible que temas muy concretos y avanzados no aparezcan, pero a grandes rasgos por lo menos todo fenómeno gramatical está explicado. Sus cinco capítulos son: una introducción, las clases de palabras, las oraciones y grupos, fonética y fonología y la ortografía, y todos contienen ejercicios con las soluciones al final del ejemplo. Esto hace que además de poder ejercer como libro de consulta pueda emplearse en clases magistrales y/o prácticas como libro de texto.

(Informante 2.42)»

Estos usos incorrectos de los signos de puntuación tienen una incidencia negativa en la correcta construcción de la oración y del párrafo y en la organización global del texto que puede afectar a la coherencia y la cohesión de todas las partes, lo cual puede llevar al lector a una interpretación diferente de la deseada.

\subsection{Prácticas escritoras}

Los resultados sobre las prácticas escritoras se sintetizan en la siguiente tabla 4, que comprende las prácticas escritoras que los docentes solicitan, con la denominación que ellos le dan, según el curso. Asimismo, se acompañan estas prácticas con un porcentaje que equivale al espacio que se le dedica a cada una en total en dicho curso.

Tabla 4.

Prácticas escritoras según el curso

\begin{tabular}{lllll}
\hline & Primero & Segundo & Tercero & Cuarto \\
\hline Examen & $15,6 \%$ & $28,5 \%$ & $33,4 \%$ & $37,1 \%$ \\
Reseña & $8,8 \%$ & $2,7 \%$ & $0 \%$ & $0 \%$ \\
Artículo periodístico & $8,8 \%$ & $0 \%$ & $0 \%$ & $0 \%$ \\
Ensayo & $7,3 \%$ & $16,6 \%$ & $25 \%$ & $28,5 \%$ \\
Comentario de texto & $4,4 \%$ & $8,3 \%$ & $0 \%$ & $4,1 \%$ \\
Resumen & $4,4 \%$ & $5,5 \%$ & $3,7 \%$ & $2 \%$ \\
& & & &
\end{tabular}




\begin{tabular}{|c|c|c|c|c|}
\hline Monografía & $2,9 \%$ & $2,7 \%$ & $3,7 \%$ & $2 \%$ \\
\hline Póster científico & $2,9 \%$ & $0 \%$ & $0 \%$ & $0 \%$ \\
\hline Ponencia & $0 \%$ & $2,7 \%$ & $3,7 \%$ & $0 \%$ \\
\hline Manual & $0 \%$ & $2,7 \%$ & $3,7 \%$ & $0 \%$ \\
\hline Trabajo fin de grado & $0 \%$ & $0 \%$ & $0 \%$ & $2 \%$ \\
\hline Esquema & $0 \%$ & $0 \%$ & $3,7 \%$ & $0 \%$ \\
\hline Entrada de blog & $0 \%$ & $5,5 \%$ & $3,7 \%$ & $0 \%$ \\
\hline Aforismo & $0 \%$ & $5,5 \%$ & $3,7 \%$ & $0 \%$ \\
\hline Glosario & $2,9 \%$ & $0 \%$ & $0 \%$ & $0 \%$ \\
\hline Definición & $2,9 \%$ & $0 \%$ & $0 \%$ & $0 \%$ \\
\hline Gráfico & $2,9 \%$ & $0 \%$ & $0 \%$ & $0 \%$ \\
\hline Power Point & $0 \%$ & $2,7 \%$ & $0 \%$ & $2 \%$ \\
\hline Currículum & $0 \%$ & $0 \%$ & $3,7 \%$ & $0 \%$ \\
\hline Carta de solicitud de trabajo & $0 \%$ & $0 \%$ & $3,7 \%$ & $0 \%$ \\
\hline Postal & $2,9 \%$ & $0 \%$ & $0 \%$ & $0 \%$ \\
\hline Correo electrónico & $2,9 \%$ & $0 \%$ & $0 \%$ & $0 \%$ \\
\hline Guion cinematográfico & $0 \%$ & $0 \%$ & $0 \%$ & $2 \%$ \\
\hline Nota & $2,9 \%$ & $0 \%$ & $0 \%$ & $0 \%$ \\
\hline Invitación & $2,9 \%$ & $0 \%$ & $0 \%$ & $0 \%$ \\
\hline Planificación de un día & $2,9 \%$ & $0 \%$ & $0 \%$ & $0 \%$ \\
\hline Cuento & $2,9 \%$ & $0 \%$ & $0 \%$ & $0 \%$ \\
\hline Narración corta & $2,9 \%$ & $0 \%$ & $0 \%$ & $0 \%$ \\
\hline Biografía & $2,9 \%$ & $0 \%$ & $0 \%$ & $0 \%$ \\
\hline Entrada de wiki & $2,9 \%$ & $0 \%$ & $0 \%$ & $0 \%$ \\
\hline Noticia & $2,9 \%$ & $0 \%$ & $0 \%$ & $0 \%$ \\
\hline Reportaje & $2,9 \%$ & $0 \%$ & $0 \%$ & $0 \%$ \\
\hline Informe & $1,4 \%$ & $0 \%$ & $8,3 \%$ & $10,2 \%$ \\
\hline Trabajo escrito & $1,4 \%$ & $16,6 \%$ & $0 \%$ & $10,2 \%$ \\
\hline
\end{tabular}

Fuente: elaboración propia.

La Tabla 4 pone de manifiesto que los estudiantes se ven envueltos en la producción de prácticas escritoras de diferentes ámbitos como pueden ser el de los medios de comunicación, la vida cotidiana, el administrativo, el literario y, por supuesto, el académico. No obstante, estas prácticas son poco exigentes desde el punto de vista discursivo y, cognitivamente, están asociadas a los saberes que les están transmitiendo, como el aprendizaje de habilidades idiomáticas (noticia, biografía, nota, correo electrónico, postal...). De hecho, la alta recurrencia en cada curso de textos cuya producción se basa en la lectura de otros textos (ensayo, reseña, comentario de texto, resumen, esquema...) y textos orientados únicamente a la evaluación (examen) infiere que la escritura de textos en el grado está, fundamentalmente, orientada a la evaluación de los conocimientos disciplinares.

La escritura que plantea retos retórico-discursivos y cognitivos para la creación de conocimiento no ocupa un lugar destacado en el grado de LLMM, con pocas excepciones como la monografía, el póster científico o la ponencia. Estos son textos más especializados y más exigentes que requieren que los estudiantes estén inmersos en el área disciplinar y se posicionen críticamente mediante el diálogo con otras voces; son más extensos y compartimentados en secciones que requieren mantener una orientación argumentativa a lo largo de un número elevado de páginas; el autor y el lector están muy presentes en dichos textos y hay unos convencionalismos propios de la disciplina y del propio contexto para dejar su huella.

Otra cuestión importante de estos resultados es la falta de progresión de la complejidad de las prácticas escritoras de un curso a otro. La alfabetización académica de los estudiantes universitarios 
es un proceso en constante evolución a medida que se presentan nuevos desafíos retóricos y discursivos, y en el que los docentes deben implicarse mediante una enseñanza de prácticas en contexto (Carlino, 2013), pero no se constata ese proceso, esa progresión, de un curso a otro.

\section{Conclusión}

En este apartado conclusivo se sintetizan los resultados más destacados en relación a los cinco objetivos de este trabajo. Empezando por la selección de la información en las reseñas, se constata que los estudiantes realizan una selección de los contenidos cada vez más pertinente de un curso a otro cuando están describiendo, pero que esta selección es, al mismo tiempo, limitada, pues no aborda con suficiente profundidad el objeto de la reseña. Por otro lado, cuando los estudiantes valoran, no se manifiesta una progresión clara en la selección de la información de un curso a otro, y se constatan las mismas limitaciones que cuando describen. Estos resultados se alinean con los derivados del siguiente objetivo del estudio, que aborda la organización de los contenidos, donde se observa, en todos los cursos por igual, preferencia por secuenciaciones discursivas que primero describen y luego valoran, como es propio de este género discursivo, pero también dificultades en la progresión temática del texto que se reflejan en la recursividad de la descripción y la valoración.

En lo que respecta a cómo configuran su propia voz como autores académicos, los resultados ponen de manifiesto que los estudiantes, a medida que pasan los cursos, asumen un papel como autores cada vez más objetivo, con posturas más neutrales, que no dialoga con otras voces. Un autor que, pese a tener presente al lector en el texto, no hace evidente la estructuración del texto para facilitar su comprensión.

En cuanto a los aspectos formales, los resultados evidencian dificultades en la acentuación de las palabras, el uso de las mayúsculas y de los signos de puntuación (en especial, la coma, el punto y coma, el punto y aparte y el punto final). Estas dificultades, presentes de manera cada vez más notoria según pasan los cursos, inciden negativamente en la fluidez de la lectura, en la comprensión de los contenidos e incluso en la organización del texto.

Por último, las prácticas escritoras que los estudiantes deben producir en el grado son, salvo unas pocas excepciones, poco exigentes cognitiva, retórica y discursivamente, y, además, no permiten la comprensión del texto como un evento comunicativo. Se da también la circunstancia de que la mayor parte de estas prácticas están dirigidas a evaluar conocimientos disciplinares relativos a objetivos educativos de un nivel de complejidad medio bajo (conocimiento-comprensión-aplicación y, quizás en algunos casos, análisis). Por otra parte, estas prácticas escritoras no se vuelven más complejas a medida que avanzan los cursos, no plantean nuevos desafíos a los estudiantes, ni permiten aprovechar todo el potencial epistémico de la escritura académica.

Estos resultados parecen alinearse con los de las producciones de los estudiantes, en los que la progresión realizada de un curso a otro es limitada o incluso no se manifiesta claramente. Por otra parte, el grado de alfabetización académica de estos estudiantes presenta una serie de déficits que anticipan que no están adecuadamente preparados para el abordaje del trabajo de fin de grado, pero que también convierten dicha tarea en una oportunidad para continuar el proceso de alfabetización académica, como ya se ha señalado en otros estudios (Rekalde, 2011; Sierra, 2016).

En definitiva, mediante este estudio se materializa la importancia de los tipos de prácticas escritoras que los estudiantes realizan y de la progresión de estas a lo largo de su trayectoria formativa para el desarrollo de la alfabetización académica. Ahora bien, estos resultados no deben extrapolarse a otras titulaciones y a otras realidades sin la debida investigación empírica que las respalde. Este estudio se ha centrado en el grado de LLMM de la Universidad de Deusto, pero un estudio más ambicioso que incorpore el análisis de más titulaciones, más géneros académicos y profesionales (dado el carácter profesionalizante de muchos grados) y más universidades podría mostrar una panorámica más precisa del grado de alfabetización de los estudiantes de grado en relación con las prácticas discursivas que estos realizan. Asimismo, los resultados obtenidos no solo plantean interrogantes sobre el desempeño de estos estudiantes para realizar una tarea como el trabajo de fin de grado, sino también sobre las dificultades en la producción de textos académicos a las que tendrán que enfrentarse durante los estudios de posgrado. 


\section{Referencias}

Aguilar, L., \& Fregoso, G. (2013). La lectura de la polifonía e intertextualidad en el texto científico. Revista Mexicana de Investigación Educativa, 18(57), pp. 413-435. Recuperado de https://ref.scielo.org/gcphs6

Alter, C., \& Adkins, C. (2006). Assessing student writing proficiency in graduate schools of social work. Journal of Social Work Education, 42(2), pp. 337-354. doi: 10.5175/JSWE.2006.200404109

Álvarez, M., \& Boillos, M. (2015). La producción escrita de los estudiantes universitarios de nuevo ingreso. Magis, Revista Internacional de Investigación en Educación, 8(16), pp. 71-90. doi: 10. 11144/Javeriana.m8-16.peeu.

Alzari, I. (2012). La reseña como género de formación en la carrera de Historia (pp. 148-154). $V$ Congreso Internacional de letras. Buenos Aires: Facultad de Filosofía y Letras de la Universidad de Buenos Aires. Recuperado de https://2012.cil.filo.uba.ar/ponencia/la-rese\%C3\%B1a-como$\mathrm{g} \% \mathrm{C} 3 \% \mathrm{~A} 9$ nero-de-formaci $\% \mathrm{C} 3 \% \mathrm{~B} 3$ n-en-la-carrera-de-historia

Alzari, I., D’Alessandro, J., \& Radiminski, M. (2014). La reseña de formación en historia. En F. Navarro, Manual de escritura para carrera de Humanidades (pp. 287-302). Buenos Aires: Facultad de Filosofía y Letras de la Universidad de Buenos Aires. Recuperado el 28 de diciembre del 2019 de https:/ /discurso.files.wordpress.com/2014/08/navarro_2014_manual-de-escritura-para-carreras-de-humanidades.pdf

Arrosi, F., Axelrud, B., D’Agostino, M., \& Eisner, L. (2003). Competencias argumentativas en alumnos universitarios. Actas del Congreso Internacional «La argumentación» (pp. 656-664). Buenos Aires: Universidad de Buenos Aires.

Bazerman, C., Little, J., Bethel, L., Chavkin, T., Fouquette, D., \& Garufis, J. (2005). Reference guide to writing across the curriculum. Indiana, IL: Parlor Press \& The WAC Clearinghouse.

Bereiter, C., \& Scardamalia, M. (1987). The psycology of written composition. Hillsdle (Estados Unidos): Erlbaum.

Camps, A., \& Castelló, M. (2013). La escritura académica en la universidad. REDU. Revista de Docencia Universitaria, 11(1), pp. 17-36. doi: 10.4995/redu.2013.5590

Camps, A., \& Millian, M. (2000). El papel de la actividad metalingüistica en el aprendizaje de la escritura. Rosario: Homo Sapiens.

Carlino, P. (2003). Alfabetización académica: un cambio necesario, algunas alternativas posibles. Educere, 6(20), pp. 409-420. Recuperado de http://ref.scielo.org/pbfwkp

Carlino, P. (2013). Alfabetización académica diez años después. Revista Mexicana de Investigación Educativa, 18(57), pp. 355-381. Recuperado de https://www.redalyc.org/pdf/140/14025774003.pdf

Castelló, M. (2009). Escribir trabajos de investigación con alumnos de grado. Textos de didáctica de la lengua y la literatura, 50, pp. 21-29.

Castelló, M., Corcelles, M., Iñesta, A., Bañales, G., \& Vega, N. (2011). La voz del autor en la escritura académica: una propuesta para su análisis. Revista Signos, 44(76), pp. 105-117. doi: 10.4067/S071809342011000200001

Castelló, M. (2015). La investigación sobre escritura académica en los procesos de enseñanzaaprendizaje en la universidad española. Cultura y Educación, 27, 465-476. doi: 10.1080/11356405.2015.1072362

Castro, M., \& Sánchez, M. (2013). La expresión de opinión en textos académicos escritos por estudiantes universitarios. Revista Mexicana de Investigación Educativa, 18(57), pp. 483-506. Recuperado de http://www.scielo.org.co/pdf/leng/v44n1/v44n1a03.pdf

Cazorla, M. C. (2011). Una aproximación a los aspectos positivos y negativos derivados de la puesta en marcha del Plan Bolonia en la universidad española. REJIE: Revista Jurídica de Investigación e Innovación Educativa, 4, pp. 91-104. Recuperado de https://hdl.handle.net/10115/5764

Chartier, A. (2004). Enseñar a leer y a escribir. Una perspectiva histórica. Méjico DF: Fondo de Cultura Económica.

Chois, P., \& Jaramillo, L. (2016). La investigación sobre la escritura en posgrado: estado del arte. Lenguaje, 44(2), pp. 227-259. doi: 10.25100/lenguaje.v44i2.4622

Colmenares, S. (2013). Prácticas de escritura académica en una universidad pública colombiana: autoría, audiencia e interacción con otras voces. Lenguaje, 41(1), pp. 201-227. Recuperado de https://ref.scielo.org/pwrf7x 
Errázuriz, M., \& Fuentes, L. (2010). Evaluación y desarrollo de una propuesta centrada en la producción de textos en estudiantes de primer año de Pedagogía en Educación General Básica. Sembrando ideas. Revista Educativa, 4, pp. 38-53.

Ezeiza, J. (2012). Bases para la evaluación del dominio de las formas disciplinares de comunicación y de los usos lingüísticos especializados en el Espacio Europeo de Educación Superior (EESS). Revista Nebrija de Lingüistica Aplicada, (12). doi: 10.26378/rnlael612191

Figueroa, R., \& Simón, J. (2011). Planificar, escribir y revisar, una metodología para la composición escrita. Una experiencia con estudiantes del Instituto Pedagógico de Caracas (IPC). Revista de Investigación, 35(73), pp. 119-147. Recuperado de https://www.redalyc.org/pdf/3761/376140388006.pdf

Flower, L., \& Hayes, J. (1977). Problem- solving strategies and the writing process. College English, 39, pp. 449-61. doi: 10.2307/375768

Gil-Salom, L., \& Soler-Monreal, C. (2014). Writer's positioning in literature reviews in English and Spanish computing doctoral thesis. Journal of English for Academic Purposes, 16, pp. 23-39. doi: 10.1016/j.jeap.2014.08.002

Gómez, L. (2011). Gramática didáctica del español. Madrid: Editorial SM.

Goethals, P., \& Delbecque, N. (2001). Personas del discurso y ‘despersonalización’. En G. Vázquez, Guía práctica del discurso académico escrito. ¿Cómo se escribe una monografía? (pp. 67-80). Madrid: Edinumen.

Guzmán-Simón, F., \& García-Jiménez, E. (2015). La alfabetización académica en la universidad. Un estudio predictivo. RELIEVE, 21(1), pp. 1-17. doi: 10.7203/relieve.21.1.5018

Guzmán-Simón, F., \& García-Jiménez, E. (2017). La alfabetización académica de los futuros maestros. Un estudio comparativo en varias universidades españolas. Revista de Investigación Educativa, 35(2), pp. 317-335. doi: 10.6018/rie.35.2.246011

Hyland, K. (2007). Disciplinary discourses: social interactions in academic writing. Michigan: Ann Arbor (University of Michigan Press).

Hymes, D. (1972). Models of the interaction of language and social life. En J. Gumperz, \& D. Hymes, Directions in sociolinguistics: the ethnography of communication (pp. 35-71). Nueva York: Holt, Rinehart y Winston.

Ivanic, R. (1998). Writing and identity. Amsterdam: John Benjamins Publishing Company. doi: $10.1075 /$ swll. 5

Marinkovich, J., \& Poblete, C. (2014). Alfabetización en escritura académica en Biología: propósitos comunicativos y niveles de alfabetización. Onomázein, 30, pp. 269-285. doi: 10.7764/onomazein. 30.20

Navarro, F., \& Abramovich, A. (2012). La reseña académica. En L. Natale, En carrera: escritura y lectura de textos académicos y profesionales (pp. 39-60). Polvorines: Universidad Nacional de General Sarmiento.

Ortíz, E. (2011). La escritura académica universitaria: estado del arte. Íkala, 16(28), pp. 17-39. Recuperado de http://ref.scielo.org/9ndpcf

Real Academia de la Lengua Española. (2010a). La ortografía de la lengua española. Madrid: Espasa.

Real Academia Española. (2010b). Nueva gramática de la lengua española. Madrid: Espasa Libros, SLU.

Rekalde, I. (2011). ¿Cómo afrontar el trabajo fin de grado? Un problema o una oportunidad para culminar con el desarrollo de las competencias. Revista Complutense de Educación, 22(2), pp. 179-193. doi: 10.5209/rev_RCED.2011.v22.n2.38488

Romero, A. N. (2019). La escritura académica de estudiantes de grado a través de sus producciones: la reseña de formación. Estudio de caso de los estudiantes de Lenguas Modernas. (Tesis doctoral). Universidad de Deusto, Bilbao.

Russell, D., Lea, M., Parker, J., Street, B., \& Donahue, T. (2009). Exploring notions of genre in "academic literacies" and "writing across the curriculum": approaches across countries and contexts. En C. Bazerman, A. Bonini, \& D. Figueiredo, Genre in a changing world (pp. 459-491). Colorado: The WAC Clearinghouse/ Parlor Press.

Sánchez-Jiménez, D. (2017). Implicación de la citación en la voz del autor en el discurso académico universitario: la memoria de máster escrita en español por estudiantes españoles y filipinos. Diálogo de la Lengua, VIII, pp. 16-36. Recuperado de https://academicworks.cuny.edu/cgi/viewcontent.cgi?article=1189\&context=ny_pubs 
Sierra, J. (2016). El trabajo de fin de grado. Una oportunidad para la investigación universitaria. En A. M. Chocrón, Calidad, docencia universitaria y encuestas: «Bolonia a coste cero» (pp. 235-240). Asociación de Mujeres Laboristas de Andalucía (AMLA): Sevilla. Recuperado de https://idus.us.es/handle/11441/45226;jsessionid= 385CAB519BB59F156F91B9D7DC91082C?.

Street, B. (1996). Academic literacies. En D. Baker, C. Fox, \& J. Clay, Challenging ways of knowing: Literacies, numeracies and sciences (pp. 101-134). Brighton: Falmer Press.

Swales, J. (1990). Genre Analysis. Cambridge: Cambridge University Press.

Swales, J. (2004). Research genres: explorations and applications. Cambridge: University of Cambridge. doi: 10.1017/CBO9781139524827

Tapia, M., Burdiles, G., \& Arancibia, B. (2003). Aplicación de una pauta diseñada para evaluar informes académicos universitarios. Revista Signos, 36(54), pp. 249-257. doi: 10.4067/S071809342003005400009

Taylor, S. (2013). What is discourse analysis? India: Bloomsbury Publishing Plc.

Venegas, R., Meza, P., \& Martínez, J. (2013). Procedimientos discursivos en la atribución del conocimiento en tesis de lingüística y filosofía en dos niveles académicos. Revista de Lingüística Teórica y Aplicada, 51(1), pp. 153-179. doi: 10.4067/S0718-48832013000100008 